\title{
Behaviors of Phase Diagrams of Ising Model with Competing Ternary and Binary Interactions on a Cayley Tree of Arbitrary Order
}

\author{
H. Akin ${ }^{a}$, N. Ganikhodjaev ${ }^{b}$, S. UGUZ ${ }^{c}$ And S. TemiR ${ }^{c}$ \\ ${ }^{a}$ Faculty of Education, Department of Mathematics, Zirve University, Gaziantep, 27260, Turkey \\ ${ }^{b}$ Department of Computational and Theoretical Sciences, Faculty of Science, IIUM, 25200 Kuantan, Malaysia \\ ${ }^{c}$ Arts and Science Faculty, Department of Mathematics, Harran University, Sanliurfa, 63120, Turkey \\ An Ising model with competing interactions has recently been studied extensively because of the appearance \\ of nontrivial magnetic orderings. In this paper, we study the phase diagrams for the Ising model on a Cayley tree \\ with competing nearest-neighbor interactions $J$ and ternary prolonged interactions $J_{t_{\mathrm{p}}}$ on a Cayley tree of arbitrary \\ order $k$ and compare with the phase diagrams obtained in Uguz et al. and Vannimenus results for the Ising model \\ on a Cayley tree with competing nearest-neighbor interactions $J$ and ternary prolonged interactions $J_{\mathrm{p}}$. For some \\ values of $k$, we obtain phase diagrams of the model. We clarify the role of order $k$ of the Cayley tree. We also plot \\ the variation of the wave vector with temperature.
}

PACS: 05.50.+q, 64.60.-i, 64.60.De

\section{Introduction}

The Ising model on a Cayley tree of order $k$ with competing interactions has recently been studied extensively because of the appearance of nontrivial magnetic orderings [1] (see also Refs. in [2]). For many problems the solution on a tree is much simpler than on a regular lattice and is equivalent to the standard Bethe-Peierls theory [3]. In recent years, investigation of phase diagrams of the Ising model has attracted increased attention [4]. Inawashiro et al. [5] independently of Vannimenus investigated the Ising model with nearest-neighbors and prolonged next-nearest-neighbors interactions on a Cayley tree, but they allowed $J_{\mathrm{p}}=J_{0}$, where $J_{0}$ is the one-level next-nearest-neighbor interaction on the Cayley tree of order two. Later Mariz et al. [6] extended these results assuming also existence of binary interaction $J_{0}$ on the Cayley tree of order 2. Recently Ganikhodjaev et al. have obtained a general result of the Vannimenus work on a Cayley tree of arbitrary finite order $k$ [1]. This work is a natural continuation of the study of the phase diagrams on Cayley tree which was investigated in $[1,2,4]$ and [7].

In this paper, using the methods in $[1,2]$ we study the phase diagrams for the Ising model on a Cayley tree with competing nearest-neighbor interactions $J$ and ternary prolonged interactions $J_{\mathrm{t}_{\mathrm{p}}}$ on a Cayley tree of arbitrary order $k$ and compare with the phase diagrams obtained by Vannimenus [2] and Uguz et al. [1] results for Ising model on a Cayley tree with competing nearest-neighbor interaction $J$ and prolonged next-nearest-neighbors in- teractions $J_{\mathrm{p}}$. For some values of $k$, we obtain phase diagrams of the model. We clarify the role of order $k$ of the Cayley tree. We also plot the variation of the wave vector with temperature.

\section{The model and basic equations}

A Cayley tree $\Gamma^{k}$ of order $k \geq 1$ is an infinite tree, i.e., a graph without cycles with exactly $k+1$ edges issuing from each vertex. Let denote the Cayley tree as $\Gamma^{k}=(V, \Lambda)$, where $V$ is the set of vertices of $\Gamma^{k}, \Lambda$ is the set of edges of $\Gamma^{k}$. For a fixed $x_{0} \in V$ we set $W_{n}=\left\{x \in V \mid d\left(x, x_{0}\right)=n\right\}$,

$$
V_{n}=\left\{x \in V \mid d\left(x, x_{0}\right) \leq n\right\}=\bigcup_{i=0}^{n} W_{i}
$$

and $L_{n}$ denotes the set of edges in $V_{n}$. The fixed vertex $x_{0}$ is called the 0 -th level and the vertices in $W_{n}$ are called the $n$-th level.

For the sake of simplicity we put $|x|=d\left(x, x_{0}\right), x \in V$. Two vertices $x$ and $y, x, y \in V$ are called nearest-neighbors if there exists an edge $l \in \Lambda$ connecting them, which is denoted by $l=\langle x, y\rangle$. The distance $d(x, y), x, y \in V$, on the Cayley tree $\Gamma^{k}$, is the number of edges in the shortest path from $x$ to $y$. Two vertices $x, y \in V$ are called the next-nearest-neighbors if $d(x, y)=2$. The next-nearest-neighbor vertices $x$ and $y$ are called prolonged next-nearest-neighbors if $|x| \neq|y|$ and is denoted by $\rangle x, y<$. The vertices $x, y, z \in V$ are called ternary prolonged neighbors, if $x \in W_{n}, y \in W_{n+1}$ and $z \in W_{n+2}$, where $n \geq 0$. We 
will denote them by $>x, y, z<$ (see Ref. 8 for details). We will consider ternary prolonged neighbors instead of prolonged next-nearest-neighbors studied in [2].

In this paper, we will consider a semi-infinite Bethe lattice $\Gamma_{+}^{k}$ of order $k$, i.e. an infinite graph without cycles with $(k+1)$ edges issuing from each vertex except for $x^{0}$ which has only $k$ edges.

For the Ising model with spin values in $\Phi=\{-1,1\}$, the relevant Hamiltonian with competing nearest-neighbor and prolonged ternary neighbor interactions has the form

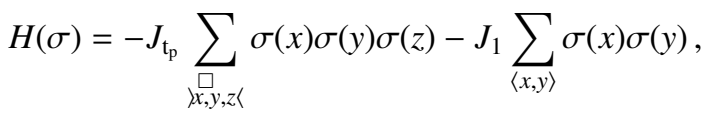

where $J_{\mathrm{t}_{\mathrm{p}}}, J_{1} \in R$ are coupling constants.

In order to produce the recurrent equations, we consider the relation of the partition function on $V_{n}$ to the partition function on subsets of $V_{n-1}$. Given the initial conditions on $V_{1}$, the recurrence equations indicate how their influence propagates down the tree. Let $Z^{(n)}\left(\begin{array}{c}i_{1}, i_{2}, \ldots, i_{k} \\ i_{0}\end{array}\right)$ be the partition function on $V_{n}$ where the spin in the root $x^{0}$ is $i_{0}$ and the $k$ spins in the proceeding ones are $i_{1}, i_{2}, \ldots, i_{k}$. There are a priori $2^{k+1}$ different $Z^{(n)}$ to consider. It is reasonable, though, to assume that the different branches are equivalent, as is usually done for models on trees. We can show that there are only four independent variables, namely $z_{1}=Z^{(n)}\left(\begin{array}{c}+,+, \ldots,+ \\ +\end{array}\right), z_{2}=Z^{(n)}\left(\begin{array}{c}-,-, \ldots,- \\ +\end{array}\right), z_{3}=$ $Z^{(n)}\left(\begin{array}{c}+,+, \ldots,+ \\ -\end{array}\right), z_{4}=Z^{(n)}\left(\begin{array}{c}-,-, \ldots,- \\ -\end{array}\right)$. Then arbitrary partial partition function $Z^{(n)}\left(\begin{array}{c}i_{1}, i_{2}, \ldots, i_{k} \\ i_{0}\end{array}\right)$ is a combination of $z_{1}, z_{2}, z_{3}, z_{4}$. For instance, for $\sigma\left(i_{0}\right)=+$ we can obtain the partial partition function as follows:

$$
Z^{(n)}\left(\begin{array}{c}
i_{1}, i_{2}, \ldots, i_{k} \\
+
\end{array}\right)=z_{1}^{\frac{m}{k}} z_{2}^{\frac{k-m}{k}}
$$

where $m, 0 \leq m \leq k$ is the number of spins up on first level $W_{1}$. Similarly, for $\sigma\left(i_{0}\right)=-$ we can obtain the partial partition function as follows:

$$
Z^{(n)}\left(\begin{array}{c}
i_{1}, i_{2}, \ldots, i_{k} \\
-
\end{array}\right)=z_{3}^{\frac{m}{k}} z_{4}^{\frac{k-m}{k}}
$$

where $m, 0 \leq m \leq k$ is the number of spins up on first level $W_{1}$. Through the introduction of the new variables $u_{i}=\sqrt[k]{z_{i}}$, we produce the following recurrence system:

$$
\begin{aligned}
& u_{1}^{\prime}=a\left(b u_{1}+b^{-1} u_{2}\right)^{k}, \quad u_{2}^{\prime}=a^{-1}\left(b^{-1} u_{3}+b u_{4}\right)^{k}, \\
& u_{3}^{\prime}=a^{-1}\left(b^{-1} u_{1}+b u_{2}\right)^{k}, \quad u_{4}^{\prime}=a\left(b u_{3}+b^{-1} u_{4}\right)^{k},
\end{aligned}
$$

where $a=\exp \left(J_{1} / T\right), b=\exp \left(J_{\mathrm{t}_{\mathrm{p}}} / T\right)$ and primed variables $u_{i}^{\prime}$ correspond to the $Z^{(n+1)}$. The total partition function is given in terms of $\left(u_{i}\right)$ by $Z^{(n)}=\left(u_{1}+u_{2}\right)^{k}+\left(u_{3}+u_{4}\right)^{k}$.

For discussing the phase diagram, the following choice of reduced variables is convenient:

$$
x=\frac{u_{2}+u_{3}}{u_{1}+u_{4}}, \quad y_{1}=\frac{u_{1}-u_{4}}{u_{1}+u_{4}}, \quad y_{2}=\frac{u_{2}-u_{3}}{u_{1}+u_{4}} .
$$

The variable $x$ is just a measure of the frustration of the nearest-neighbor bonds and is not an order parameter like $y_{1}, y_{2}$ (see [2] for details). The relations now have the following form:

$$
\begin{aligned}
x^{\prime} & =\frac{1}{a^{2} D}\left\{\left[x-y_{2}+b^{2}\left(1-y_{1}\right)\right]^{k}\right. \\
& \left.+\left[1+y_{1}+b^{2}\left(x+y_{2}\right)\right]^{k}\right\}, \\
y_{1}^{\prime} & =\frac{1}{D}\left\{\left[b^{2}\left(1+y_{1}\right)+x+y_{2}\right]^{k}\right. \\
& \left.-\left[b^{2}\left(x-y_{2}\right)+1-y_{1}\right]^{k}\right\}, \\
y_{2}^{\prime} & =\frac{1}{a^{2} D}\left\{\left[x-y_{2}+b^{2}\left(1-y_{1}\right)\right]^{k}\right. \\
& \left.-\left[1+y_{1}+b^{2}\left(x+y_{2}\right)\right]^{k}\right\},
\end{aligned}
$$

where $D=\left[x+y_{2}+b^{2}\left(1+y_{1}\right)\right]^{k}+\left[b^{2}\left(x-y_{2}\right)+1-y_{1}\right]^{k}$.

\section{Phase diagrams}

For the sake of completeness we will restate the method studied in [2]. It is necessary to know the broad features of the phase. This can be achieved numerically similar to [2]. The recursion relations (2) provide us the numerically exact phase diagram in $\left(-J_{\mathrm{t}_{\mathrm{p}}} / J_{1}, T / J_{1}\right)$ space. Assume $\left(-J_{\mathrm{t}_{\mathrm{p}}} / J_{1}\right)=\beta, T / J_{1}=\alpha$ and respectively $b=\exp \left(-\alpha^{-1} \beta\right), a=\exp \left(\alpha^{-1}\right)$. Starting from the following initial conditions:

$$
\begin{aligned}
& x^{(1)}=\frac{b^{2 k}+a^{2 k}}{a\left[(a b)^{2 k}+1\right]}, \\
& y_{1}^{(1)}=\frac{(a b)^{2 k}-1}{(a b)^{2 k}+1}, \quad y_{2}^{(1)}=\frac{b^{2 k}-a^{2 k}}{a^{2}\left[(a b)^{2 k}+1\right]},
\end{aligned}
$$

that corresponds to boundary condition $\bar{\sigma}^{(n)}\left(V \backslash V_{n}\right) \equiv 1$, one iterates the recurrence relations (2) and observes their behavior after a large number of iterations. In the simplest situation a fixed point $\left(x^{*}, y_{1}^{*}, y_{2}^{*}\right)$ is reached. It corresponds to a paramagnetic phase if $y_{1}^{*}=0, y_{2}^{*}=0$ or to a ferromagnetic phase if $y_{1}^{*}, y_{2}^{*} \neq 0$. From the formula of average magnetization (3) follows that a situation where $y_{1}^{*}, y_{2}^{*} \neq 0$ but $m=0$ cannot occur.

Secondary, the system may be periodic with period $p$, where case $p=2$ corresponds to antiferromagnetic phase and case $p=4$ corresponds to so-called antiphase. Finally, the system may remain aperiodic. The distinction between a truly aperiodic case and one with a very long period is difficult to make numerically. Below we consider periodic phases with period $p$ where $p \leq 12$. All periodic phases with period $p>12$ and aperiodic phase will be considered as modulated phase. The resultant phase diagrams for some values of $k$ are shown in Fig. 1. 


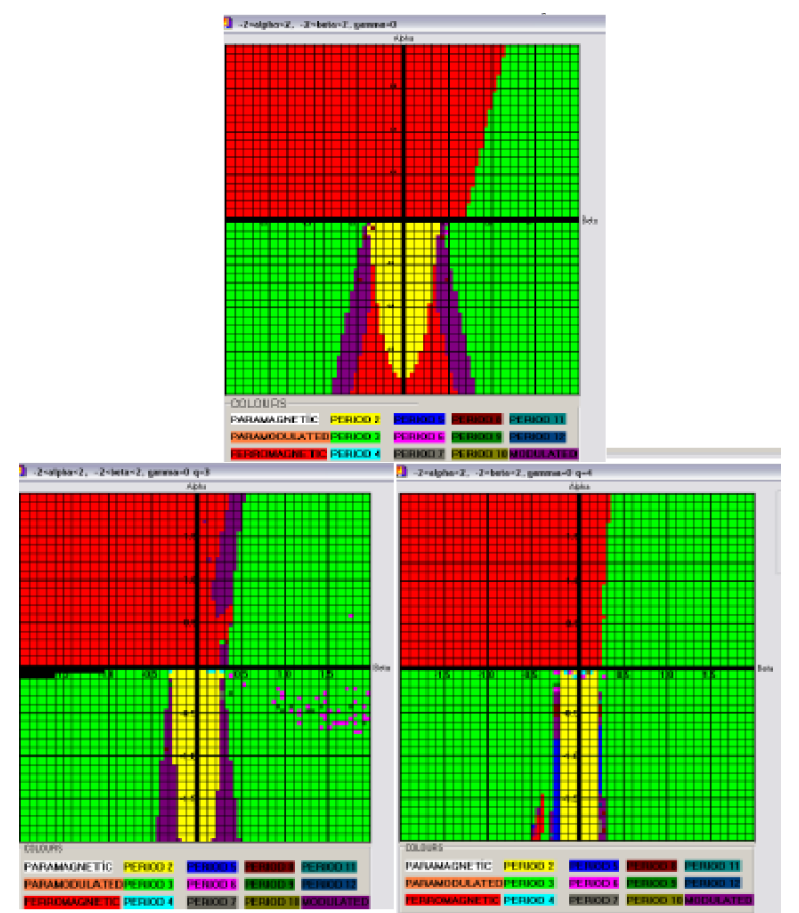

Fig. 1. Phase diagram of the model for $k=2,3,4$, respectively.

For $J_{1}>0$ and $k=2$ the phase diagram contains ferromagnetic phase and phase with period 3 , that is new (see [8]). The interesting part of phase diagram corresponds to competing interactions $\left(J_{1}<0\right)$. In this case the phase diagram is very rich and there are at least four multicritical Lifshitz points, where two of them are at zero temperature and other two are at nonzero temperature. The main novelty lies in existing multicritical Lifshitz points that are at nonzero temperature, since in previous works [2] and [6] such point was at zero temperature. Let us note that in this case paramagnetic phase is absent.

In this paper we clarify the role of order $k$ of the Cayley tree. In [6] the authors assumed that effects for $k=2$ should not be very different in higher (but finite) order $k$. However from considerations above one can see that the role of $k$ is rather significant. Secondary, for isomorphism between the system characterized by $\left(J_{1}, J_{\mathrm{t}_{\mathrm{p}}}\right)$ and that characterized by $\left(-J_{1}, J_{\mathrm{t}_{\mathrm{p}}}\right)$ stated in [6], there are small islands in the quadrant $\{\beta>0, \alpha<0\}$ where this isomorphism is broken, moreover the number of such islands increases with increasing $k$. To investigate the nature of such islands is rather a complete problem.

From consideration above we can suppose that for large $k$ a phase diagram has following structure: in first and third quadrants we have ferromagnetic and P3 phase only, in second quadrant we have ferromagnetic phase only and in third quadrant we have mainly P3 phase and a lot of islands of other phases.

Remark. Under numerical analysis provided above for some values of parameters $\alpha$ and $\beta$ we can reach very large numbers such that a computer cannot operate with them.

\section{The average magnetization}

In this section, we will study the behavior of the average magnetization in a period or the dynamic magnetization as a function of the reduced temperature. This investigation leads us to characterize the nature of the transition.
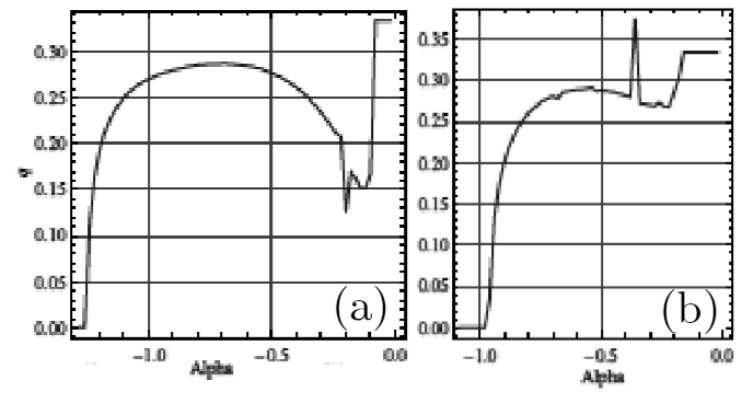

Fig. 2. Variation of the wave vector of the model for $k=2$ and (a) $\beta=-0.45$, (b) $\beta=0.47$, respectively.

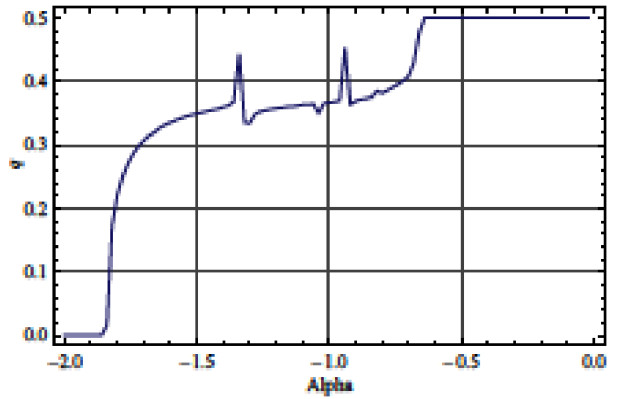

Fig. 3. Variation of the wave vector of the model for $k=3$ and $\beta=-0.27$.

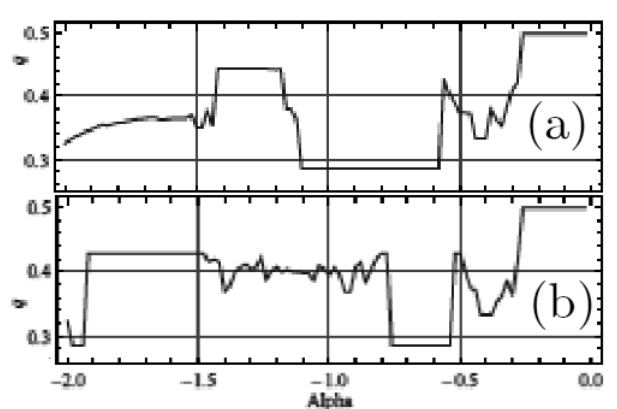

Fig. 4. The average magnetization versus $T / J$ of the model for $k=4$ and (a) $\beta=0.23$, (b) $\beta=-0.23$, respectively.

The average magnetization $m$ for the $n$-th generation is given by 


$$
m=\frac{\left(1+x+y_{1}+y_{2}\right)^{k}-\left(1+x-y_{1}-y_{2}\right)^{k}}{\left(1+x+y_{1}+y_{2}\right)^{k}+\left(1+x-y_{1}-y_{2}\right)^{k}} .
$$

Here we use numerical methods to study the behaviors of the system given in Eq. (2). Lastly we consider the variation of the wave vector with temperature. A definition of the wavevector that is convenient for numerical purposes is $q=\lim _{N \rightarrow \infty}\left(\frac{1}{2} \frac{n}{N}\right)$, where $n$ is the number of times the magnetization (3) changes sign during $N$ successive iterations [2]. The magnetization wave vector varies continuously with temperature and order of the Cayley tree. Typical graphs of $q$ versus $T$ are drawn in Figs. 2-4.

In Fig. 1 , for $k=2$ the point $(\beta, \alpha)=(-0.45,-0.15)$ is the multicritical Lifshitz one. The graphs of $q$ versus $T / J_{1}$ are changing very fast around $(-1.25,0)$. In Fig. 1, for $k=4$ in the fourth region, there is modulated phase and the island-shaped region is quite narrow. The analysis of such general phase diagrams is quite complicated. Therefore, the Lyapunov exponents need to be examined in detail. Also, for a more detailed study of variation of the wavevector $q$ versus $T / J_{1}$, we need to locate the main locking steps that should be present regarding to the general theory [2]. Because the intervals are very narrow, description of the distinction between long-periodic cycles and calculations are difficult. The answer to the our problem consists of nonlinear analysis techniques such as the Lyapunov exponents associated with the trajectory of the dynamical system (see [2]).

\section{Conclusions}

We have seen that ternary prolonged interaction has strong effects on the phase diagrams. As the first effect, they shift the multicritical Lifshitz point from zero temperature to a finite positive temperature. As the second effect, the paramagnetic phase disappears at finite temperature, while the Ising models in [1] and [2] do not possess this property. As stated in [9], the stabil- ity analysis for the ferromagnetic phase is more difficult than the stability analysis for the paramagnetic phase because the fixed point is not the same for the whole phase. The stability analysis of the ferromagnetic phase will be studied in detail in the future research paper. As stated earlier analytical investigation on the stability of fixed points (paramagnetic, ferromagnetic and modulated) given in Eqs. (2) is planned to another publication. Also, the transition lines between some new phases will also be considered later on. For particular values of the coupling constants and $T$, we have plotted the magnetization graphs of the nonlinear-dynamical system.

\section{Acknowledgments}

The work is supported by The Scientific and Technological Research Council of Turkey-TUBITAK (project No. 109T678).

\section{References}

[1] S. Uguz, N.N. Ganikhodjaev, S. Temir, H. Akın, H., AIP Conf. Proc. 1281, 2074 (2010).

[2] J. Vannimenus, Z. Phys. B 43, 141 (1981).

[3] S. Katsura, M. Takizawa, Prog. Theor. Phys. 51, 82 (1974).

[4] S. Uguz, H. Akin, Physica A 389, 1839 (2010).

[5] S. Inawashiro, C.J. Thompson, Phys. Lett. 97A, 245 (1983).

[6] M. Mariz, C. Tsalis, A.L. Albuquerque, J. Statist. Phys. 40, 577 (1985).

[7] S. Uguz, H. Akin, Chinese J. Phys. 49, 785 (2011).

[8] N.N. Ganikhodjaev, H. Akin, S. Uguz, S. Temir, Phase Transitions: A Multinational Journal 84, 1045 (2011).

[9] K. Fesser, H.J. Herrmann, J. Phys. A 17, 1493 (1984). 